\title{
Pion Corrections in Gribov's Approach to the Dyson-Schwinger Equation
}

\author{
Carlo Ewerz \\ Università di Milano and INFN, Via Celoria 16, I-20133 Milano, Italy
}

\begin{abstract}
.
Chiral symmetry breaking in QCD leads to the emergence of pions as Goldstone bosons. Their existence in turn affects the Green functions of the theory. Here we study the effect of pion corrections on the light quark's Green function in Feynman gauge using the framework of Gribov's approach to the Dyson-Schwinger equation.
\end{abstract}

\section{GRIBOV'S APPROACH}

The system of Dyson-Schwinger equations (DSEs) for the Green functions is one of the few tools we have for investigating the nonperturbative structure of QCD. In particular, one would like to use the DSEs to understand the mechanism giving rise to chiral symmetry breaking $(\chi \mathrm{SB})$ and confinement. The difficulty in doing so lies in the fact that the DSEs form a tower of coupled integral equations. Their treatment hence requires truncations or approximations which are in general difficult to control.

Some time ago Gribov suggested a new approach to the DSE for light quarks [1]. His approach is designed to systematically collect the most important contributions to this integral equation originating from the infrared (IR) region in which the dynamics leading to $\chi \mathrm{SB}$ and confinement is expected to take place. The largest contributions to the integrals in the DSE from the IR can be most conveniently identified in Feynman gauge. In that gauge the gluon propagator has the form $D_{\mu v}(k)=-\alpha_{s}(k) g_{\mu v} / k^{2}$, which can be understood as a definition of a nonperturbative coupling $\alpha_{s}(k)$ at small momenta. The applicability of Gribov's approach requires only mild assumptions on the behavior of $\alpha_{s}(k)$, namely that is does not diverge at $k=0$ and does not vary too rapidly with $k$. One then takes the second derivative of the inverse Green function, $\partial^{\mu} \partial_{\mu} G^{-1}(q)$ with $\partial_{\mu}=\partial / \partial q^{\mu}$. Using the DSE one finds that the most singular contribution from the IR comes from differentiating twice the gluon Green function because $\partial^{2}\left(1 / q^{2}\right) \sim \delta\left(q^{2}\right)$. With the help of Ward identities one then obtains a differential equation for the light quark's Green function, see eq. (1) below without the last term. It can also be shown to reproduce the correct renormalization group equation in the ultraviolet (UV) region. Less IR-singular terms can be computed systematically in this approach as subleading corrections. In this sense Gribov's approach is an approximation rather than a mere truncation scheme for the quark's DSE.

One can then use Gribov's equation for a detailed investigation of the light quark's Green function in Feynman gauge. According to [1] it should in particular be possible 
to use this approach in order to study the analytic properties of the Green function and to relate them to a picture in which confinement is caused by the phenomenon of supercritical charges. For a recent review of the ideas underlying Gribov's picture of confinement we refer the reader to [2].

\section{PIONS FROM CHIRAL SYMMETRY BREAKING}

As was discussed at a previous edition of this conference [3], in Gribov's approach it is found that $\chi \mathrm{SB}$ takes place if the strong coupling $\alpha_{s}$ exceeds a critical value of $\alpha_{c}=0.43$ in some region of momenta in the IR. In this situation the dynamical mass function $M\left(q^{2}\right)$ exhibits oscillations around zero, and as a consequence the relation between the perturbative (or current) quark mass and the renormalized mass $m_{R}=M(0)$ is no longer one-to-one. Instead one finds that a nonvanishing renormalized mass can be generated even for vanishing perturbative quark mass. It was found though that in the approximation discussed above the analytic structure of the Green function does not correspond to confined quarks [4].

When $\chi \mathrm{SB}$ takes place massless pions are created as Goldstone bosons and appear in the physical spectrum. Their Bethe-Salpeter amplitude can be obtained as $\varphi \sim\left\{i \gamma_{5}, G^{-1}\right\}$ from an equation for $q \bar{q}$ bound states derived in the same approximation as discussed above for the quark [1].

In the phase of $\chi \mathrm{SB}$ in which pions exist as (massless) physical particles it is natural to consider their backreaction on the quark's Green function. In the first approximation described above their effect is not properly taken into account. Instead, they have to be included explicitly. It was argued in [5] that pion corrections can have a crucial effect in particular on the analytic structure of the Green function, possibly giving rise to the confinement of quarks.

\section{PION EFFECTS ON THE QUARK'S GREEN FUNCTION}

It turns out that the emission and reabsorption of pions can be included very easily in Gribov's approximation scheme for the quark DSE. The pion propagator $\sim 1 / k^{2}$ in connection with the differential operator $\partial^{2}$ makes it again possible to isolate the most important IR contribution in diagrams with pion loops on the quark. Further, we express the Bethe-Salpeter amplitude of the pion in terms of $G$, as a result of which we are again left with a differential equation for $G$ only. Finally, the pion-quark coupling can be fixed with the help of the Goldberger-Treiman relation. The resulting equation for $G(q)$ reads

$$
\partial^{\mu} \partial_{\mu} G^{-1}=\frac{C_{F} \alpha_{s}}{\pi}\left(\partial^{\mu} G^{-1}\right) G \partial_{\mu} G^{-1}-\frac{3}{16 \pi^{2} f_{\pi}^{2}}\left\{i \gamma_{5}, G^{-1}\right\} G\left\{i \gamma_{5}, G^{-1}\right\}
$$

where the last term constitutes the modification of the original equation due to pions.

We have performed a numerical study of this improved equation for the Green function of light quarks. Here we concentrate an the dynamical mass function $M$ in the euclidean region, $q^{2}<0$. More detailed results will be presented elsewhere. 
Recall that the Green function can be written as $G^{-1}=Z^{-1}(\not 1-M)$ with the wave function renormalization factor $Z^{-1}$. From equation (1) one finds analytically that the pion corrections become negligible in the UV region. Hence also the modified equation reproduces the correct RG behavior there. Figure 1 shows our numerical results for the mass function. They have been obtained with an IR frozen coupling $\alpha_{s}(q)$, see [4]. The
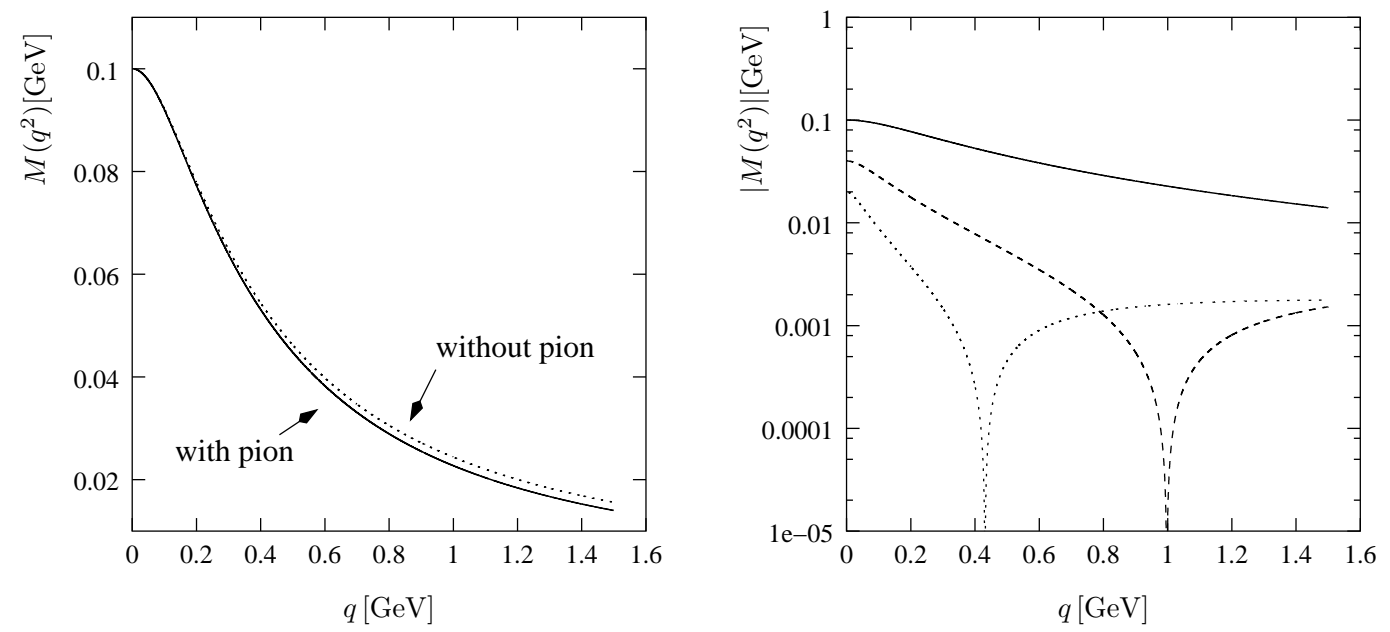

FIGURE 1. Change in the mass function due to pion corrections (left), and the mass function for three different values of the renormalized mass (right)

mass function is affected only very little due to the pion corrections (see example on the left). The figure on the right shows $M\left(q^{2}\right)$ for different values of the renormalized mass. For sufficiently small $m_{R}$ the mass function oscillates, giving rise to $\chi \mathrm{SB}$.

In summary, we find only small effects of the pion corrections for spacelike momenta. The pattern of chiral symmetry breaking is the same as in the approximation without pions. Preliminary results indicate that the analytic structure of the quark's Green function in the complex $q^{2}$-plane, on the other hand, is considerably changed due to the pion corrections, as was anticipated in [5]. This interesting aspect and its potential consequences for confinement clearly deserve further study.

\section{ACKNOWLEDGMENTS}

This work was supported by a Feodor Lynen fellowship of the Alexander von Humboldt Foundation.

\section{REFERENCES}

1. Gribov, V. N., Eur. Phys. J., C10, 71-90 (1999).

2. Dokshitzer, Y. L., and Kharzeev, D. E., hep-ph/0404216 (2004).

3. Ewerz, C., "Gribov's Light Quark Confinement Scenario," in Quark Confinement and the Hadron Spectrum V, edited by N. Brambilla and G. Prosperi, World Scientific, 2003, pp. 309-311.

4. Ewerz, C., Eur. Phys. J., C13, 503-518 (2000).

5. Gribov, V. N., Eur. Phys. J., C10, 91-105 (1999). 